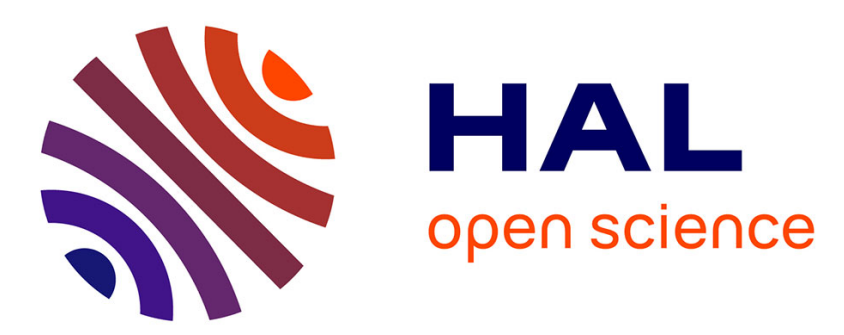

\title{
APPLICATION DE L'ANALYSE EN ONDELETTES À LA DÉTECTION DE FISSURES PAR ÉCHOGRAPHIE ULTRASONORE
}

J. Lefebvre, P. Lasaygues

\section{- To cite this version:}

J. Lefebvre, P. Lasaygues. APPLICATION DE L'ANALYSE EN ONDELETTES À LA DÉTECTION DE FISSURES PAR ÉCHOGRAPHIE ULTRASONORE. Journal de Physique IV Proceedings, 1992, 02 (C1), pp.C1-637-C1-640. 10.1051/jp4:19921137 • jpa-00251094

\author{
HAL Id: jpa-00251094 \\ https://hal.science/jpa-00251094
}

Submitted on 1 Jan 1992

HAL is a multi-disciplinary open access archive for the deposit and dissemination of scientific research documents, whether they are published or not. The documents may come from teaching and research institutions in France or abroad, or from public or private research centers.
L'archive ouverte pluridisciplinaire HAL, est destinée au dépôt et à la diffusion de documents scientifiques de niveau recherche, publiés ou non, émanant des établissements d'enseignement et de recherche français ou étrangers, des laboratoires publics ou privés. 


\title{
APPLICATION DE L'ANALYSE EN ONDELETTES À LA DETECTION DE FISSURES PAR ÉCHOGRAPHIE ULTRASONORE
}

\author{
J.P. LEFEBVRE et P. LASAYGUES
}

Laboratoire de Mécanique et d'Acoustique, 31 Ch. Joseph-Aiguier, F-13402 Marseille cedex 9, France

\begin{abstract}
This paper is related to the problem of echographic detection of cracks under coatings. It is concerned with both modelization and signal processing. For the modelization of scattering, we have chosen monoscattering approximations (Born for interfaces in material, Physical Optics for cracks) that appear to be very efficient for the problem. As a tool for signal processing, we have chosen a time-scale method, the wavelet analysis, that appears to be very well suited for signal discrimination.
\end{abstract}

\section{Introduction}

L'échographie est la technique de base du Contrôle Non Destructif par ultrasons. Elle consiste à exploiter les échos provenant des diverses inhomogénéités rencontrées par l'onde. Deux phénomènes extrêmes jouent un rôle important dans la formation des échos: les réflexions, dues aux objets de grande dimension devant la longueur d'onde (interfaces,...) et les diffusions provoquées par les objets de petite dimension devant la longueur d'onde (inclusions,...). On montre que les signaux de réflexion sont en gros des copies du signal émis par le transducteur, et les signaux de diffusion des copies de la dérivée seconde de ce signal (1). On peut donc s'attendre à ce que les objets de dimension intermédiaire, comme les têtes de fissure, donnent des signatures intermédiaires. C'est effectivement ce qu'on constate expérimentalement: un écho d'inclusion est sensiblement la dérivée d'un écho de tête de fissure, et lui-même donc la dérivée d'un écho d'interface. D'où la méthode de discrimination de signaux de fissures (2) qui consiste à apprécier si un signal échographique est proche d'un écho de diffusion (défaut ponctuel - faible nocivité) ou de son intégrale (bord de fissure - forte nocivité).

Le but de la présente étude est d'une part de fournir un fondement théorique aussi simple que possible à cette méthode de discrimination, d'autre part de la doter d'un outil de traitement du signal adapté. Pour le premier point nous avons choisi les approximations de l'optique physique et de Born qui sont un bon compromis simplicité-qualité. Pour le second point nous avons choisi une technique temps-échelle, l'analyse en ondelettes, l'idée étant de discriminer les signatures dans le plan spectral tout en conservant une bonne localisation temporelle, c'est à dire spatial puisqu'il s'agit d'échographie.

\section{Modélisation}

On s'intéresse à la diffraction d'une impulsion ultrasonore large bande par une fissure. Dans le cadre de l'approximation de Born (obstacle pénétrable), ou celle de l'Optique Physique (obstacle impénétrable), on montre que la réponse impulsionnelle en rétrodiffusion à grande distance $\mathrm{HR}_{\mathrm{R}}(\mathrm{t})$ est proportionnelle à la dérivée seconde de la fonction d'aire $\mathrm{A}(\mathrm{x})$ de l'obstacle dans la direction Ox d'émission-réception considérée, avec le changement de variable espace-temps de parcours $x=\frac{c t}{2}$ :

$$
H_{R}(t)=-\frac{c}{8 \pi}\left(\frac{d^{2} A(x)}{d x^{2}}\right)_{x=\frac{c t}{2}}
$$


Cette identité est connue en électromagnétisme sous le nom d'identité de Bojarski (3). Son inversion est l'identité de POFFIS (Physical Optics Far Field Inverse Scattering), utilisée en radar pour déterminer la fonction d'aire d'un obstacle.

En échographie, le même transducteur est utilisé, en régime impulsionnel, à la fois comme émetteur et comme récepteur, de sorte que le signal recueuilli est la convolution de la réponse impulsionnelle en rétrodiffusion du milieu avec la réponse impulsionnelle en réflexion de la chaine, définie comme la réponse à un réflecteur plan parfait normal.

Si nous modélisons la fissure par un demi-plan réfléchissant (condition de Dirichlet), sa fonction d'aire est un échelon de Heaviside et sa dérivée seconde la dérivée d'un "Dirac". La réponse impulsionnelle en rétrodiffusion de la fissure est donc la dérivée d'un "Dirac" et sa convolution avec la réponse impulsionnelle en réflexion de la chaîne la dérivée de cette dernière, c'est à dire la dérivée d'un écho d'interface normal. C'est bien le résultat attendu.

\section{Traitement des signaux}

Nous avons choisi l'analyse en ondelettes, plus précisément l'analyse surabondante (4) qui présente à la fois les avantages de l'analyse continue (présentation des résultats sous forme d'images facilitant les analyses) et de l'analyse discrète (orthogonalité des voies entières).

Cette analyse consiste à associer à un signal $S(t)$ la suite:

$$
\chi^{\mathrm{a}, \mathrm{b}}=\int_{-\infty}^{\infty} \Psi^{\mathrm{a}, \mathrm{b}}(\mathrm{t}) S(\mathrm{t}) d \mathrm{t},
$$

où $\Psi a, b(t)=\sqrt{a} \Psi\left[a\left(t-\frac{b}{a}\right)\right]$ est une version comprimée (du facteur a) et translatée (de la quantité $\frac{b}{a}$ ) de l'ondelette analysante $\Psi(t)$.

L'ondelette analysante $\Psi(\mathrm{t})$ que nous avons choisie est celle de Meyer-Jaffard (5). Son spectre est centré $\operatorname{sur} \frac{2}{3} U_{f}$ ( $U_{f}$ étant une unité fréquentielle de réfèrence, en pratique la fréquence d'échantillonnage $F e$ ), a une largeur de bande totale de 2 octaves et une largeur de bande à -3 dB de 1 octave. Dans le domaine temporel, elle est réelle et inférieure à $10^{-3}$ en dehors d'un intervalle de longueur $13 U_{t}$ (où $U_{t}=U_{f^{-1}}$ ) centré sur $\frac{1}{2} \mathrm{U}_{\mathrm{t}}$.

A cette ondelette mère, on associe la famille d'ondelettes $\Psi_{j, k}\left(a=2 j\right.$ et $b=k U_{t}$, avec $j$ et $k$ entiers relatifs) définie par :

$$
\Psi \mathrm{j}, \mathrm{k}(\mathrm{t})=\sqrt{2 j} \Psi\left[2 \mathrm{j}\left(\mathrm{t}-\frac{\mathrm{k}}{2 j} \mathrm{U}_{\mathrm{t}}\right)\right]
$$

Cette famille constitue une base orthogonale sur laquelle on peut développer toute fonction réelle de $t$.

De par sa définition, l'ondelette $\Psi_{\mathrm{j}, \mathrm{k}}(\mathrm{t})$ est essentiellement concentrée autour de $\mathrm{t}=2^{-j}\left(\mathrm{t}-\frac{1}{2}\right) U_{\mathrm{t}}$ tandis que son spectre est maximum à la fréquence $v^{0} \mathrm{j}=\frac{2 \mathrm{j}+1}{3} U_{f}$ et a pour support l'intervalle:

$\left[\frac{2 j}{3} U_{f}, \frac{2 j+2}{3} U_{f}\right]$. Elle effectue donc une analyse localisée à la fois en temps et en fréquence.

Tout le problème réside dans le choix des paramètres de dilatation-compression $\mathrm{j}$ et de translation $\mathrm{k}$. On traite le cas d'un signal $s(t)$ échantillonné, comportant $N$ points. On montre que si on choisit comme unité de référence fréquentielle $U_{f}$ la fréquence d'échantillonnage $\mathrm{Fe}$ du signal $S$, les seules voies non nulles sont les voies $\mathrm{j} \leq 0$ et donc on ne fait finalement que des dilatations de l'ondelette analysante. Par ailleurs, on a toujours affaire à des signaux suffisamment sur-échantillonnés pour que le spectre du signal soit quasi nul au delà de $\frac{F_{\mathrm{e}}}{3}$. La voie $\mathrm{j}=0$ n'est donc généralement pas calculée non plus.

Les coefficients d'ondelettes associés à une fonction $S(t)$ sont définis comme les projections de $S(t)$ sur cette base orthogonale. On peut aussi les interpréter comme l'intercorrélation entre le signal $S(t)$ et l'ondelette centrée à la voie $j$, prise aux points $t j, k=\frac{k}{2 j} U_{t}$ : 


$$
\chi_{j, k}=\int_{-\infty}^{\infty} \Psi_{j, k}(t) S(t) d t=\left[S * \Psi^{\#}, 0\right]\left(\frac{k}{2 j}-U_{t}\right)
$$

On peut étendre les définitions précedentes au cas de voies non entières, en particuklier fractionnaires (de $j \in Z$ on passe à $\gamma \in Q$ ). On parle alors d'analyse surabondante. L'orthogonalité ne concerne plus que les points situés sur la grille dyadique. Dans ce cas, on préfère utiliser les ondelettes analytiques associées aux ondelettes de départ. Lorsqu'on calcule un grand nombre de voies intermédiaires (entre les voies entières), on obtient un résultat comparable à celui que donne l'analyse continue (résultats présentés sous forme d'image). On parle alors d'analyse pseudo-continue. Nous avons mis au point des logiciels rapides à base de FFT et les avons utilisés d'une part comme outil de discrimination entre signature d'interface fissuré et signature d'interface sain (comparaison entre les deux signatures), d'autre part comme aide pour évaluer la qualité des modélisations proposées (comparaisons signaux expérimentaux- simulations).

\section{Application à une fissure sous revêtement}

On s'est intéressé au problème industriel de la détection d'une fissure dans une pièce massive $(10 \mathrm{~cm}$ d'épaisseur) en acier munie d'un revêtement épais $(1 \mathrm{~cm})$ en acier inoxydable. Ce revêtement étant obtenu par soudure de couches successives, l'interface est plus ou moins plan mais son contraste est très faible (impédances acoustiques des deux aciers très voisines) de sorte que l'approximation de Born se justifie. La fissure, lorsqu'elle existe, présente la particulariré de déboucher le plus souvent à l'interface (c'est alors qu'elle est la plus nocive) perpendiculairement à lui, de sorte qu'en inspection normale l'écho de tête de fissure est confondu avec celui de l'interface et donc complètement masqué. Le problème n'est donc pas simplement de discriminer un écho de fissure d'un écho d'interface, mais un écho d'interface fissuré d'un écho d'interface sain. En faisant l'hypothèse apparamment réaliste de monodiffusion (échos très faibles) le problème de diffraction est linéarisé, et on obtient la réponse en diffraction de l'interface fissuré par simple superposition des réponses de l'interface et de la fissure.

\section{Conclusion}

Les résultats obtenus révèlent une bonne concordance théorie-expérience pour les signatures échographiques tant de la fissure seule que de l'interface fissuré. La concordance est bonne sur les signaux bruts ainsi que sur leurs analyses en ondelettes. La modélisation proposée est donc très satisfaisante pour l'ingénieur, en ce sens qu'elle est à la fois simple et efficace.

Ces résultats révèlent également la bonne qualité de l'outil "ondelettes", adapté aussi bien à la validation de modèles (confers supra ) qu'à la discrimination de signatures et donc à l'analyse de signaux de contrôle non destructif par ultrasons. A titre d'exemple, les figures 1 et 2 montrent comment l'analyse en ondelettes révèle bien les différences de signature entre un interface sain et un interface fissuré. Les différences sont notables tant sur l'analyse pseudo-continue (figure 1: grand nombre de voies intermédiaires calculées, présentation de l'analyse sous forme d'image comme en analyse continue) que sur l'analyse discrète (figure 2: petit nombre de voies intermédiaires calculées, ici 1 et présentation de l'analyse sous forme de réseau de courbes). Notons au passage que l'analyse othogonale stricte n'est pas adaptée à l'analyse de ce type de signature (les voies entières ne sont pas discriminantes).

\section{Bibliographie}

(1) P.M. Morse, K.U. Ingard, Theoretical acoustics, Mc Mraw-Hill Book company, NY 1968.

(2) D. De Vadder, M. Dosso, Caractérisation ultrasonore des bords de fissure par traitement numérique du signal, Proc. 3rd. Europ. Conf. Nondestructive Testing, Firenze (I), t.5, pp.362-374, 1984.

(3) N. Bojarski, A survey of the Physical Optics Inverse Scattering Identity, IEEE Trans. Antennas and Propagation, vol. AP-30, $\mathrm{n}^{\circ} 5$, pp. 980-989, 1982.

(4) V. Perier, C. Basdevant, La décomposition en ondelettes périodiques, un outil pour l'analyse des champs inhomogènes, La Recherche Aérospatiale, $n^{\circ} 1989-3$, pp. 53-67, 1989.

(5) S. Jaffard, Algorithmes de transformation en ondelettes, Rapport Ecole Polytechnique - ENPC, 1987. 


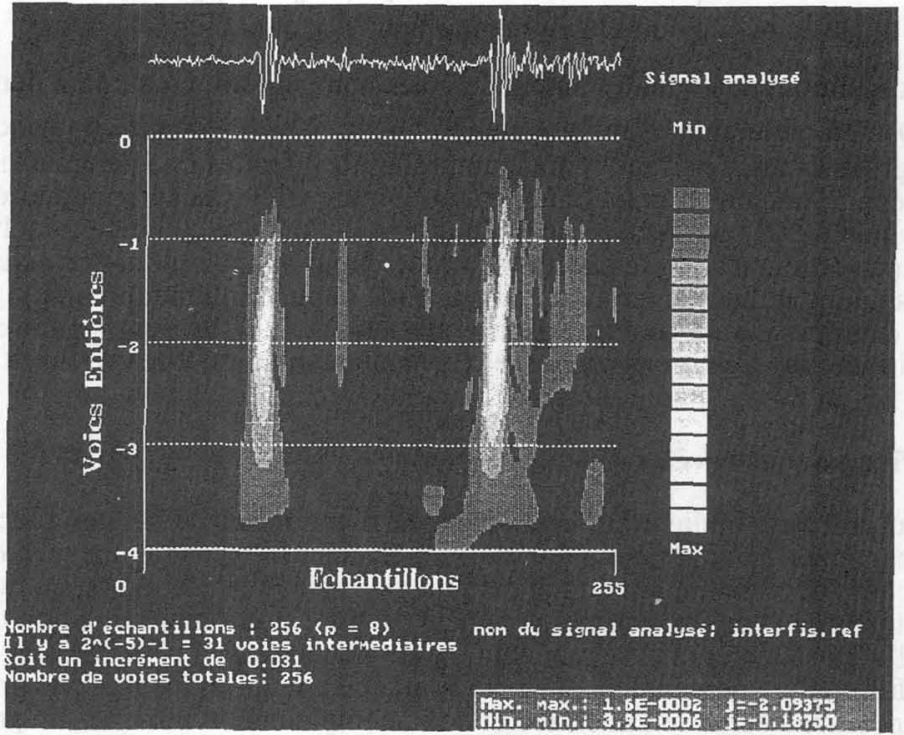

Figure 1. Détection de fissure par analyse en ondelettes (analyse pseudo-continue)

à gauche: interface sain

à droite: interface fissuré

(on remarque la fourche, caractéristique de linterface fissuré)

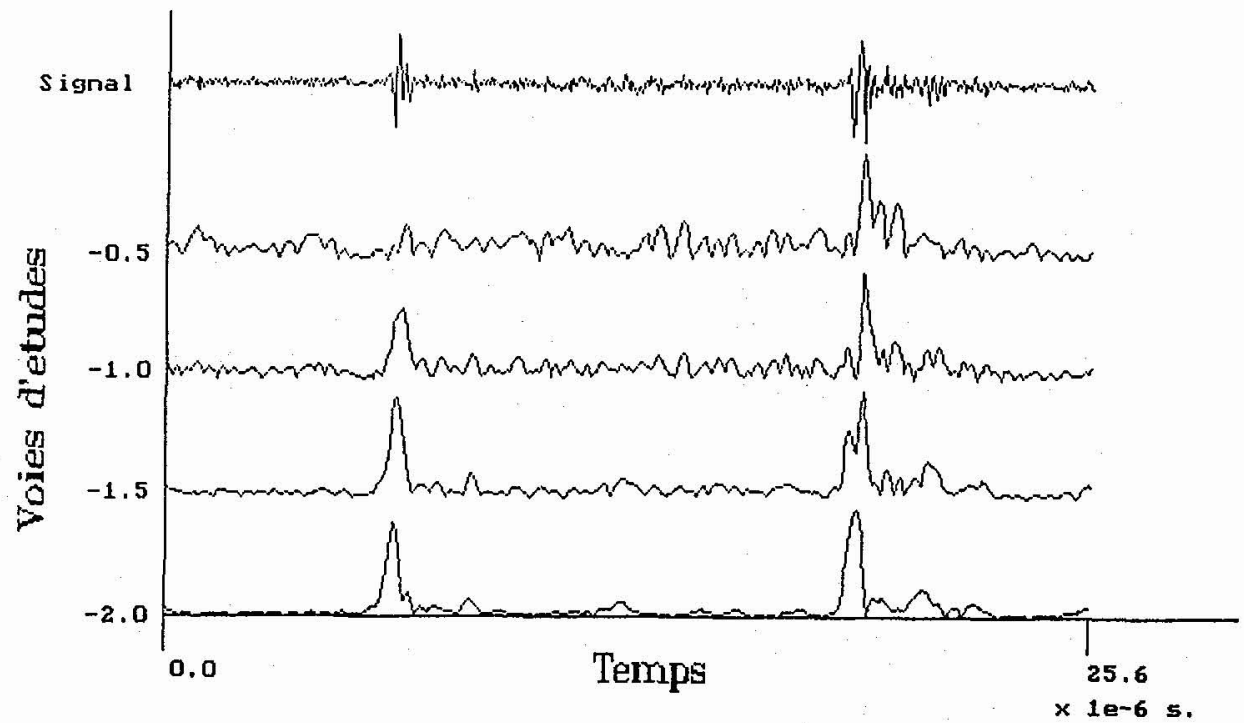

Figure 2. Détection de fissure par analyse en ondelettes (analyse discrète) à gauche: interface sain à droite: interface fissuré

(sur la voie -1/2 le premier signal a disparu et le second non; sur la voie $-3 / 2$ le second est dédoublé et le premier non) 